\title{
Boson-fermion unification, superstrings, and Bohmian mechanics
}

\author{
Hrvoje Nikolić \\ Theoretical Physics Division, Rudjer Bošković Institute, \\ P.O.B. 180, HR-10002 Zagreb, Croatia \\ e-mail: hrvoje@thphys.irb.hr
}

June 12, 2009

\begin{abstract}
Bosonic and fermionic particle currents can be introduced in a more unified way, with the cost of introducing a preferred spacetime foliation. Such a unified treatment of bosons and fermions naturally emerges from an analogous superstring current, showing that the preferred spacetime foliation appears only at the level of effective field theory, not at the fundamental superstring level. The existence of the preferred spacetime foliation allows an objective definition of particles associated with quantum field theory in curved spacetime. Such an objective definition of particles makes the Bohmian interpretation of particle quantum mechanics more appealing. The superstring current allows a consistent Bohmian interpretation of superstrings themselves, including a Bohmian description of string creation and destruction in terms of string splitting. The Bohmian equations of motion and the corresponding probabilistic predictions are fully relativistic covariant and do not depend on the preferred foliation.
\end{abstract}

PACS: 03.65.Pm, 11.25.-w, 03.65.Ta

Keywords: Boson; fermion; unification; superstring; Bohmian mechanics

\section{Introduction}

All known elementary particles belong to one of the two fundamentally different types: bosons and fermions. The unification of these two types of particles is one of the most fundamental issues in elementary-particle physics. The best known theoretical principle for the unification of bosons and fermions is supersymmetry (see, e.g., [1]). In particular, supersymmetric field theories are invariant with respect to a transformation that mixes bosonic and fermionic fields. Nevertheless, in field theories, each particle species is described by a different field. Consequently, in supersymmetric field theories, bosonic and fermionic fields are still different fields. Some supersymmetric field theories (typically, theories with only one supersymmetric charge) can be formulated in terms of superfields that 
describe both bosonic and fermionic fields as parts of a single superfield, but a superfield formulation does not exist for all supersymmetric field theories.

Different particle species are truly unified in string theories [2, 3, 4]. In string theory, different particle species correspond to different states of a single object - the string. Consequently, in supersymmetric string theory (shortly - superstring theory), bosons and fermions correspond to different states of a single string.

One of the differences between bosonic and fermionic particles is the fact that bosonic particles are described by second-order differential equations (e.g., Klein-Gordon equation), whereas fermionic particles are described by first-order differential equations (e.g., Dirac equation). Consequently, the properties of the associated conserved particle current $j^{\mu}(x)$ are substantially different for bosons and fermions. The most notable difference is the fact that $j^{0}(x)$ cannot be negative for fermions, whereas it can be negative (as well as positive) for bosons (see, e.g., [5]).

In contrast to bosonic and fermionic particles, a superstring satisfies a set of equations called super-Virasoro constraints that, in particular, contains stringy analogs of both the Klein-Gordon equation and the Dirac equation. In this paper we exploit this unifying feature of bosons and fermions to introduce a new, more unified, theoretical framework to describe some aspects of bosons and fermions. More precisely, we introduce (i) particle currents for bosons and fermions in a more symmetric manner, and (ii) a superstring current from which these bosonic and fermionic particle currents can be derived.

In construction of these particle and superstring currents we get confronted with two seemingly obscure features. First, it turns out that the symmetric treatment of bosonic and fermionic particle currents requires an introduction of a preferred foliation of spacetime. It seems surprising that a requirement of one symmetry (a symmetry between bosons and fermions) breaks another symmetry (a spacetime symmetry). Is there any reasonable physical interpretation of such a preferred foliation of spacetime? Second, the physical meaning of the particle and superstring currents themselves seems obscure too. What is the physical interpretation of these currents? In this paper we argue that these seemingly obscure features acquire a natural interpretation in the context of Bohmian interpretation of particles and strings. To explain it, let us introduce the reader to the subject by shortly explaining what the significance of the Bohmian interpretation is, what the main problems with this interpretation are, and how the results of this paper help in solving them.

The Bohmian interpretation [6, 7, 8, 9, 10] is the best known and most successful attempt to introduce a hidden-variable completion of quantum mechanics (QM). According to this interpretation, particle positions or fields have a continuous and deterministic dependence on time even when they are not measured, while all quantum uncertainties emerge from an ignorance of the actual initial conditions. This interpretation offers a solution to the measurement problem of quantum mechanics, but leads to several problems that make this interpretation not widely accepted.

First, owing to the Bell theorem [11], any hidden-variable completion of quantum mechanics must be nonlocal, which is usually considered unacceptable in particle physics and field theory. However, string theory contains certain nonlocal properties that have no analog in particle physics and field theory (see, e.g., [12, 13]), so, as observed in [14], nonlocality does not seem to be an unacceptable feature from the point of view of string theory. Moreover, the nonlocal string Bohmian equation of motion seems to be a natural 
consequence of the world-sheet covariance of string theory [15], which is an argument for the Bohmian interpretation that has no analog in particle physics.

Second, in the Bohmian interpretation of nonstring theories, it is not clear whether the fundamental objects are particles or fields. Owing to the fundamental difference between bosonic and fermionic particle currents discussed above, as well as owing to the obscure nature of Grassmann-valued fields, it is often argued that bosons are fields, whereas fermions are particles [9, 10]. Another boson-fermion asymmetric Bohmian interpretation proposes that only bosonic fields have a true objective existence [16], so that all measurements eventually reduce to a detection of bosons (e.g., photons). However, such boson-fermion asymmetric interpretations are clearly incompatible with superstring theory. In this paper we formulate the Bohmian interpretation of superstrings, which in the low-energy limit necessarily leads to a boson-fermion symmetric Bohmian interpretation of bosonic and fermionic particles. Thus, superstring theory supports a picture according to which, at low energies, fundamental objects are bosonic and fermionic particles, rather than fields.

Perhaps the most undesirable feature of the Bohmian interpretation is the fact that all attempts to reconcile nonlocality of the Bohmian interpretation with the requirement of relativistic covariance lead, in one way or another, to a preferred foliation of spacetime (see, e.g., [9, 10, 17, 18, 19, 20, 21, 22]). Typically, a need for a preferred foliation occurs because one needs to specify a "time coordinate" with respect to which the nonlocal Bohmian influences between spatially separated degrees of freedom are instantaneous. On the other hand, most other interpretations of quantum mechanics (which, of course, have their own problems not shared by the Bohmian interpretation) do not need such a preferred foliation. Therefore, the Bohmian interpretation would be much more appealing if there were an interpretation-independent theoretical argument for the existence of a preferred foliation, or if it could be reformulated without need for a preferred foliation. In a sense, in this paper we do both. First, we argue that it is precisely the requirement of boson-fermion unification of the particle currents that provides such a missing argument for the existence of a preferred foliation, which then makes the Bohmian interpretation more appealing as well. Second, we formulate the Bohmian interpretation in such a manner that it does not explicitly need a preferred foliation. Instead, the preferred foliation is only needed to make an objective notion of particles consistent with quantum field theory (QFT) in curved spacetime, because otherwise particles are not well defined objects from the point of view of QFT in curved spacetime [23].

The paper is organized as follows. In the next section we study bosonic and fermionic particle currents and use heuristic arguments (based on the assumption of boson-fermion unification) to construct a new fermionic particle current that leads to a preferred spacetime foliation in effective field theory. In Sec. 3 we introduce a natural superstring generalization of the particle current from which the unified bosonic and fermionic particle currents can be derived. Sec. 4 is devoted to the Bohmian interpretation, in which these particle and superstring currents attain a natural interpretation. In Sec. 5 we study the probabilistic interpretation of nonrelativistic and relativistic wave functions and show that it is consistent with the deterministic Bohmian interpretation. The conclusions are drawn in Sec. 6.

In the paper, we use the spacetime signature $(+,-, \cdots,-)$, while the units are chosen so that $\hbar=c=1$. 


\section{Particle currents for bosons and fermions}

\subsection{The standard approach}

A prototype of a wave equation for a bosonic particle is the Klein-Gordon equation

$$
\left(\partial^{\mu} \partial_{\mu}+m^{2}\right) \varphi=0 .
$$

(Unless stated otherwise, in this section we assume that the number of spacetime dimensions is $D=4$.) It describes particles with spin 0 . The associated conserved current is

$$
j_{\mu}=i \varphi^{*} \stackrel{\leftrightarrow}{\partial_{\mu}} \varphi,
$$

which is conserved in the sense that

$$
\partial_{\mu} j^{\mu}=0 .
$$

We see that the Klein-Gordon equation is a second-order equation, and consequently, that the conserved current contains the first derivatives of $\varphi$. We also see that $j^{0}$ is not necessarily positive. Even if $\varphi$ is a superposition of plane waves with positive frequencies only, $j^{0}(x)$ may still be negative locally. In particular, it implies that $j^{0}(x)$ cannot be interpreted as a relativistic probability density.

A prototype of a wave equation for a fermionic particle is the Dirac equation

$$
\left(i \gamma^{\mu} \partial_{\mu}-m\right) \varphi=0 .
$$

It describes particles with spin $\frac{1}{2}$. Here $\gamma^{\mu}$ are matrices that satisfy the Clifford algebra

$$
\left\{\gamma^{\mu}, \gamma^{\nu}\right\}=2 \eta^{\mu \nu},
$$

where $\eta^{\mu \nu}=\operatorname{diag}(1,-1,-1,-1)$ is the Minkowski metric. They also have the property $\gamma^{0 \dagger}=\gamma^{0}, \gamma^{i \dagger}=-\gamma^{i}$, for $i=1,2,3$. The wave function $\varphi$ is a 4 -component spinor. The algebra (5) implies that $\varphi$ in (4) also satisfies the Klein-Gordon equation (11). On the other hand, a solution of the Klein-Gordon equation (11) does not necessarily need to satisfy the Dirac equation (4). Consequently, from solutions of (44) one can construct a different conserved current that, in general, cannot be constructed from solutions of (11). This current naturally associated with (4) is the Dirac current

$$
j_{\mathrm{D}}^{\mu}=\bar{\varphi} \gamma^{\mu} \varphi,
$$

where $\bar{\varphi} \equiv \varphi^{\dagger} \gamma^{0}$. It can be shown (see, e.g., [5]) that (6) transforms as a vector, and similarly, that $\bar{\varphi} \varphi$ transforms as a scalar. We see that the Dirac equation (4) is a first-order equation, and consequently, that the conserved current (6) does not contain derivatives of $\varphi$. Eq. (5) implies $\gamma^{0} \gamma^{0}=1$, so (6) implies $j_{\mathrm{D}}^{0}=\varphi^{\dagger} \varphi \geq 0$. In particular, it implies that $j_{\mathrm{D}}^{0}(x)$ as given by (6) can, potentially, be interpreted as a relativistic probability density. (This, of course, does not prove that such an interpretation is physically correct.)

The moral of this subsection is that, in the standard approach, there is a large difference between bosonic and fermionic wave functions and associated conserved currents. In 
particular, whereas the quantity $j_{\mathrm{D}}^{0}$ can, potentially, be interpreted as a probability density for the fermionic case, such an interpretation of $j^{0}$ does not work in the bosonic case. Of course, when $\varphi$ is interpreted as a bosonic or a fermionic quantum field (see, e.g., [24]), then such a probabilistic interpretation of $\varphi$ is no longer an issue. However, as reviewed in [25], quantum field theory (QFT) does not completely solve the problem, because it does not explain why the "first-quantized" probabilistic interpretation of $\varphi^{*}(x) \varphi(x)$ is physically correct in the nonrelativistic limit. Moreover, from the string-theory perspective, it is possible that string field theory is not the correct approach to treat the string interactions [26], which implies that, at low energies, particles may be more fundamental objects than fields. Thus, the goal of this paper is to introduce currents that treat bosons and fermions in a more symmetric way and to give an appropriate physical interpretation of such currents.

\subsection{The Duffin-Kemmer approach}

To make boson and fermion currents more similar, it has been proposed to replace the Klein-Gordon equation for spin-0 particles with a first-order equation similar to the Dirac equation. Such a first-order equation describing massive spin-0 particles is known as the Duffin-Kemmer equation [27]

$$
\left(i \beta^{\mu} \partial_{\mu}-m\right) \Psi=0,
$$

where $\beta^{\mu}$ are matrices that satisfy

$$
\beta^{\mu} \beta^{\nu} \beta^{\lambda}+\beta^{\lambda} \beta^{\nu} \beta^{\mu}=\beta^{\mu} \eta^{\nu \lambda}+\beta^{\lambda} \eta^{\nu \mu} .
$$

The wave function $\Psi$ has 5 components that are related to the Klein-Gordon wave function $\varphi$ satisfying (11) via

$$
\Psi=\frac{1}{\sqrt{m}}\left(\begin{array}{c}
\partial_{\mu} \varphi \\
m \varphi
\end{array}\right) .
$$

The appropriate conserved current has been constructed in [28]. (For a review with an application to the Bohmian interpretation, see also [29].) One first constructs the conserved energy-momentum tensor

$$
\Theta^{\mu \nu}=m \bar{\Psi}\left(\beta^{\mu} \beta^{\nu}+\beta^{\nu} \beta^{\mu}-\eta^{\mu \nu}\right) \Psi,
$$

where $\bar{\Psi} \equiv \Psi^{\dagger}\left(2\left(\beta^{0}\right)^{2}-1\right)$. The conserved current is then the Duffin-Kemmer current

$$
j_{\mathrm{DK}}^{\mu}=\Theta^{\mu \nu} n_{\nu},
$$

where $n^{\mu}$ is a unit future-oriented timelike vector. In particular, by taking $n^{\mu}=(1,0,0,0)$, one finds $j_{\mathrm{DK}}^{0}=\Theta^{00}=m \Psi^{\dagger} \Psi \geq 0$, which, potentially, may be interpreted as a probability density. However, a price to be paid is that it is necessary to introduce a special timelike direction determined by the vector $n^{\mu}$. This direction defines a preferred foliation of spacetime.

We also note that bosonic and fermionic currents are still quite different, as the fermionic current (6) is not constructed from the energy-momentum tensor, whereas the bosonic current (11) is. In fact, as we shall see in Sec. 3, the Duffin-Kemmer approach reviewed above does not seem to be supported by superstring theory. Therefore, in the next subsection, we propose a different approach. 


\subsection{A bosonlike approach}

In the preceding subsection, we have reviewed an approach that attempts to modify the bosonic current in a manner that makes it more similar to the standard fermionic current. One of the consequences is a need to introduce a preferred foliation of spacetime. In this subsection we propose a reversed strategy: we modify the fermionic current in a manner that makes it more similar to the standard bosonic current. It will turn out that a need for an introduction of a preferred foliation of spacetime will emerge again. However, such a unified treatment of bosonic and fermionic currents will turn out to be supported by superstring theory.

Consider a spin- $\frac{1}{2}$ wave function $\varphi$ that satisfies the Dirac equation (4). There are 4 "natural" (but different) currentlike quantities that can be constructed from $\varphi$ :

$$
\begin{array}{cl}
\bar{\varphi} \gamma_{\mu} \varphi, & \varphi^{\dagger} \gamma_{\mu} \varphi, \\
i \bar{\varphi} \overleftrightarrow{\partial_{\mu}} \varphi, & i \varphi^{\dagger} \overleftrightarrow{\partial_{\mu}} \varphi
\end{array}
$$

Let us shortly discuss their properties. The first current $\bar{\varphi} \gamma_{\mu} \varphi$ is the standard fermionic current (6) . It is conserved, real, and transforms as a vector. The second current $\varphi^{\dagger} \gamma_{\mu} \varphi$ is not conserved, the space components are not real, and it does not transform as a vector. The third current $i \bar{\varphi} \overleftrightarrow{\partial}_{\mu} \varphi$, just as the standard one, is conserved, real, and transforms as a vector. The last current $i \varphi^{\dagger} \overleftrightarrow{\partial}_{\mu} \varphi$ is conserved and real, but it does not transform as a vector. So, which current is the correct one? Since our main guiding principle is the unification of bosons and fermions, the most natural choice is the last one

$$
j_{\mu}=i \varphi^{\dagger} \stackrel{\leftrightarrow}{\partial_{\mu}} \varphi
$$

because only this current has the same form as the bosonic current (2). More precisely, (13) looks just like a current of a many-component spin-0 wave. Therefore, we propose that (13) is the correct current for fermionic particles. Indeed, as we shall see in Sec. 3, such a unified particle current for bosons and fermions naturally emerges from superstring theory. Therefore, for the sake of an easier comparison with superstrings, we also write (13) in a slightly different form. The spinor components $\varphi_{m}$ can be represented by $\varphi(m)=$ $\sum_{m^{\prime}} \delta\left(m-m^{\prime}\right) \varphi_{m^{\prime}}$, which allows to view the label $m$ as a continuous label and, up to an inessential normalization factor, to write (13) as

$$
j_{\mu}(x)=i \int d m \varphi^{*}(x, m) \overleftrightarrow{\partial_{\mu}} \varphi(x, m)
$$

Eq. (14) will turn out to have a natural generalization in superstring theory.

The problem with our choice (13) is that this current does not transform as a vector. Nevertheless, this problem can be avoided by writing it as

$$
j_{\mu}=i \bar{\varphi} \gamma^{0} \overleftrightarrow{\partial}_{\mu} \varphi=i \bar{\varphi}\left(\gamma^{\nu} n_{\nu}\right) \overleftrightarrow{\partial_{\mu}} \varphi
$$

where $n^{\mu}=(1,0,0,0)$. From the last expression in (15), we see that this current transforms as a vector. However, the price paid is that it is necessary to introduce a preferred foliation of spacetime induced by the unit future-oriented timelike vector $n^{\mu}$. The physical meaning of such a preferred foliation is discussed in the next subsection. 


\subsection{Preferred foliation and particles vs fields}

It is interesting to observe that both attempts (the one introduced in the preceding subsection as well as the one reviewed in Sec. 2.2) to introduce particle currents for bosons and fermions in a more similar way led to a preferred foliation of spacetime. Does this fact bring a deeper physical message? In this subsection we propose an answer to this question and support it by some quantitative and qualitative arguments.

In Eq. (15), the need for an introduction of a preferred foliation of spacetime emerged from the observation that otherwise the current (13) does not transform as a vector. But why exactly (13) does not transform as a vector? This is because $\varphi$ is a spinor (not merely a collection of scalars) that transforms nontrivially under Lorentz transformations. But why must $\varphi$ transform in such a nontrivial way? This transformation is derived [5] from the requirement that the Dirac equation (44) should be covariant. However, the crucial assumption in this derivation is that $\gamma^{\mu}$ is not a vector, but a collection of fixed matrices.

Conversely, if $\gamma^{\mu}$ were assumed to be a vector, then the covariance of (44) would be consistent with the possibility that $\varphi$ does not transform as a spinor, but merely as a collection of Lorentz scalars. (This should not be confused with a more common approach based on vielbeins [3, 23], in which $\gamma^{\mu}$ transforms as a vector under general coordinate transformations. In this picture, $\varphi$ transforms as a spinor under internal Lorentz transformations, which does not comply with our unifying picture of bosons and fermions. The relation between these two pictures will become clearer in Sec. 3.) In this case, the current (13) would be a genuine vector without the need for an introduction of a preferred foliation of spacetime. Could such a reinterpretation of the current (13) be the correct one? As we shall see in Sec. 3, in superstring theory (which, indeed, provides an explicit unification of bosons and fermions) $\gamma^{\mu}$ really emerges from a vector quantity and $\varphi$ really emerges from a quantity that transforms as a Lorentz scalar. Thus, from the fundamental superstring point of view, the current (13) is naturally interpreted as a genuine vector.

Now, with such a reinterpretation of $\gamma^{\mu}$ and $\varphi$, it is the Dirac current (6)

$$
j_{\mathrm{D}}^{\mu}=\varphi^{\dagger} \gamma^{0} \gamma^{\mu} \varphi
$$

that does not transform as a vector, unless a preferred foliation with the normal $n^{\mu}$ is used, so that we can write

$$
j_{\mathrm{D}}^{\mu}=\varphi^{\dagger}\left(\gamma^{\nu} n_{\nu}\right) \gamma^{\mu} \varphi .
$$

Does the fact that now (16) does not transform as a vector represent a physical problem? For free particles, there are no substantial problems. For example, the Dirac equation can be derived from a scalar Lagrangian density $\varphi^{\dagger}\left(i \gamma^{\mu} \partial_{\mu}-m\right) \varphi$, while the Dirac current (16) does not play a particular role for free particles. However, the Dirac current plays an important role for particle interactions. For example, the coupling between a charged fermion and the electromagnetic field $A_{\mu}$ is given by the interaction Lagrangian proportional to $A_{\mu} j_{\mathrm{D}}^{\mu}$, which now is not a scalar, unless a preferred foliation is introduced. This means that a true problem occurs only when one attempts to describe particle interactions. However, from the fundamental superstring point of view, this does not represent a problem at all because, in perturbative superstring theory, all interactions are described by the free superstring Lagrangian [3, 4]. Thus, the problem occurs only at the level of low-energy effective QFT description of interactions. 
The observations above also lead to an interesting reinterpretation of the theory of quantum particles and fields in classical curved backgrounds. In the usual interpretation of QFT in curved spacetime [23], the fundamental quantity is assumed to be a quantum field operator $\hat{\phi}(x)$, while the concept of a particle is regarded as emergent. It turns out that the notion of particles depends on the choice of the time coordinate with respect to which the notion of positive and negative frequencies is defined. In other words, in general, the notion of a particle is not well defined. An alternative is to introduce a preferred foliation of spacetime, which then allows to introduce a unique notion of particles described by local covariant field operators of particle currents [30]. The point is that, when the field is assumed to be fundamental, then it is the particle (not the field) that is problematic and requires a preferred foliation. On the other hand, if one assumes that the fundamental quantity is not a field but a superstring, then the low-energy object described by the fundamental quantity is a particle, not a field. (Of course, if the fundamental quantity at high energies is not a string but a string field, then the conclusion above changes. Here, however, we consider the possibility that string field theory is not the correct approach to treat strings at a nonperturbative level [26].) In other words, from the point of view of the fundamental perturbative superstring theory, at low energies particles are more fundamental objects than fields, while fields only serve as auxiliary mathematical objects useful for a description of particle interactions at low energies. This means that in curved spacetime particles should be well defined even without a preferred foliation, while it is the concept of an associated quantum field that is not well defined unless a preferred foliation is introduced. Indeed, such a reinterpretation of QFT in curved spacetime is consistent with our conclusion above that a need for a preferred foliation in the Dirac current (17) occurs only at the level of low-energy effective QFT description of interactions.

To further clarify the conceptual difference between quantum particles and fields, consider a free hermitian quantum field operator $\hat{\phi}(x)$ satisfying the Klein-Gordon equation (1). To attribute a particle interpretation to the field $\hat{\phi}(x)$, one is forced to expand it as

$$
\hat{\phi}(x)=\sum_{k} \hat{a}_{k} \varphi_{k}(x)+\hat{a}_{k}^{\dagger} \varphi_{k}^{*}(x)
$$

where the functions $\varphi_{k}(x)$ and $\varphi_{k}^{*}(x)$ have positive and negative norms, respectively, and constitute some complete orthonormal set of solutions to (1). In the particle interpretation, the operators $\hat{a}_{k}$ and $\hat{a}_{k}^{\dagger}$ are interpreted as the destruction and creation operators, respectively. However, in a curved-spacetime generalization of (11), the choice of the basis $\left\{\varphi_{k}, \varphi_{k}^{*}\right\}$ is not unique, which implies that the definition of particles based on the destruction and creation operators $\hat{a}_{k}$ and $\hat{a}_{k}^{\dagger}$ is also not unique. In particular, if $|\Phi\rangle$ is a QFT state, then the positive-norm 1-particle wave function is [31]

$$
\varphi(x)=\langle 0|\hat{\phi}(x)| \Phi\rangle,
$$

where $|0\rangle$ is the vacuum defined by $\hat{a}_{k}|0\rangle=0$. (For a generalization to the many-particle wave function and spin $\frac{1}{2}$, see also [32, 19, 20].) Thus, the 1-particle wave function $\varphi(x)$ is also not unique. In the conventional interpretation, the field $\hat{\phi}(x)$ is, by assumption, always well defined, so it is the particle wave function $\varphi(x)$ on the left-hand side of (19) that cannot be defined without a preferred foliation of spacetime. However, in our reinterpretation, fields and wave functions exchange their roles. Now the particle wave 
function $\varphi(x)$ is assumed to be fundamental and well defined. The field then only plays an auxiliary role in writing the wave function in terms of fields as in (19). To do this, one is forced to expand the positive-norm 1-particle wave function $\varphi(x)$ as

$$
\varphi(x)=\sum_{k} c_{k} \varphi_{k}(x)
$$

where $c_{k}$ are some complex coefficients, so that one can define the auxiliary field operator through the expansion (18) that allows the identification (19). Now it is the field operator (18) that depends on the choice of the basis $\left\{\varphi_{k}, \varphi_{k}^{*}\right\}$, while the expansion (20) of the wave function is considered purely conventional. (We shall discuss it more systematically elsewhere.) Although we are not able to prove explicitly that the preferred foliation needed for the unification of bosonic and fermionic currents must necessarily coincide with the preferred foliation needed for the relation between particles and fields, the simplest possibility is, indeed, that these two foliations coincide.

To summarize, we conjecture that the preferred foliation that emerges from our attempt to introduce a unifying current for bosons and fermions is a manifestation of the fact that a QFT description of particle interactions is not fundamental. The implications on the theory of particles and fields in curved spacetime will be studied in detail elsewhere. The fundamental physical origin of this preferred foliation is still unclear, but the sections that follow provide a further insight. In the next section we show how the unifying current appears more naturally in superstring theory, while in Sec. 4 we propose an interpretation of the particle and superstring currents.

\section{Superstring current}

\subsection{Elements of superstring theory}

To make this paper accessible to readers not familiar with superstring theory, following [3], in this subsection we briefly outline some basics of superstring theory. The shape of a classical string evolving in spacetime is described by real functions $X^{\mu}(\sigma, \tau), \mu=$ $0,1, \ldots, D-1$, where $\sigma \in[0, \pi]$ is the parameter along the string, and $\tau$ parametrizes time. A superstring is also equipped by Grassmann-valued coordinates $\psi^{\mu}(\sigma, \tau)$, where each $\psi^{\mu}$ is a 2-component world-sheet Majorana spinor

$$
\psi^{\mu}=\left(\begin{array}{c}
\psi_{1}^{\mu} \\
\psi_{2}^{\mu}
\end{array}\right) \equiv\left(\begin{array}{c}
\psi_{-}^{\mu} \\
\psi_{+}^{\mu}
\end{array}\right) .
$$

In addition, $\psi^{\mu}$ transforms as a vector with respect to Lorentz transformations in spacetime. (Our convention for various types of indices is summarized in Table 1.) Introducing the notation $\tau=\sigma^{0}, \sigma=\sigma^{1}$, the action of a superstring can be written as

$$
A=-\frac{1}{2} \int d^{2} \sigma\left[\left(\partial_{\alpha} X^{\mu}\right)\left(\partial^{\alpha} X_{\mu}\right)+i \bar{\psi}^{\mu} \rho^{\alpha} \partial_{\alpha} \psi_{\mu}\right]
$$

where $\alpha=0,1, \rho^{\alpha}$ are $2 \times 2$ matrices

$$
\rho^{0}=\left(\begin{array}{cc}
0 & -i \\
i & 0
\end{array}\right), \quad \rho^{1}=\left(\begin{array}{cc}
0 & i \\
i & 0
\end{array}\right),
$$




\begin{tabular}{|c|c|c|}
\hline manifold & vector indices & spinor indices \\
\hline spacetime & $\mu, \nu$ & $m$ \\
world-sheet & $\alpha, \beta$ & $b$ \\
\hline
\end{tabular}

Table 1: Summary of the indices conventions.

satisfying the 2-dimensional Clifford algebra $\left\{\rho^{\alpha}, \rho^{\beta}\right\}=2 \eta^{\alpha \beta}$, and $\bar{\psi} \equiv \psi^{\dagger} \rho^{0}$. This action is invariant under the global world-sheet supersymmetry transformations

$$
\begin{gathered}
\delta \psi^{\mu}=-i\left(\partial_{\alpha} X^{\mu}\right) \rho^{\alpha} \epsilon, \quad \delta \bar{\psi}^{\mu}=i\left(\partial_{\alpha} X^{\mu}\right) \bar{\epsilon} \rho^{\alpha}, \\
\delta X^{\mu}=\bar{\epsilon} \psi^{\mu},
\end{gathered}
$$

where $\epsilon$ is a constant infinitesimal Grassmann-valued 2-component Majorana spinor.

When $\epsilon$ is not constant, then (22) is no longer invariant under (24). Instead, the variation of the action $\delta A$ is proportional to $\int d^{2} \sigma\left(\partial_{\alpha} \bar{\epsilon}\right) S^{\alpha}$, where

$$
S_{\alpha}=\frac{1}{2} \rho^{\beta} \rho_{\alpha} \psi^{\mu} \partial_{\beta} X_{\mu}
$$

is the supercurrent. (In the literature, $S_{\alpha}$ is usually denoted by $J_{\alpha}$, but we change the notation because in this paper we will introduce a new superstring current $J_{\mu}$ that generalizes the particle current $j_{\mu}$ of Sec. 2.) In superstring theory, one actually requires local (not only global) world-sheet supersymmetry. This means that $S_{\alpha}$ should vanish for physical states. Similarly, the requirement that the action should be invariant under reparametrizations of the world-sheet coordinates $\sigma^{\alpha}$ implies that the world-sheet energymomentum tensor $T_{\alpha \beta}$ should also vanish for physical states. (To understand this, note that in arbitrary world-sheet coordinates the Minkowski metric $\eta_{\alpha \beta}$ generalizes to a more general metric $h_{\alpha \beta}\left(\sigma^{0}, \sigma^{1}\right)$, while the measure $d^{2} \sigma$ generalizes to $d^{2} \sigma \sqrt{|h|}$, where $h$ is the determinant of $h_{\alpha \beta}$. Since the action (22) generalized in this way must be reparametrization invariant, we must have $\delta A / \delta h_{\alpha \beta}=0$. This implies that the energy-momentum tensor defined as $T^{\alpha \beta}=(-2 / \sqrt{|h|}) \delta A / \delta h_{\alpha \beta}$ must vanish.) It is convenient to introduce the light-cone world-sheet coordinates $\sigma^{ \pm}=(\tau \pm \sigma)$, so that the constraints on $S_{\alpha}$ and $T_{\alpha \beta}$ can be written as

$$
T_{++}=T_{--}=0, \quad S_{+}=S_{-}=0 .
$$

From these local constraints one can construct an infinite set of global constraints. For open strings, these global constraints are

$$
L_{n}=0, \quad F_{l}=0,
$$

where

$$
\begin{aligned}
L_{n} & \equiv \int_{0}^{\pi} d \sigma\left(e^{i n \sigma} T_{++}+e^{-i n \sigma} T_{--}\right), \\
F_{l} & \equiv \sqrt{2} \int_{0}^{\pi} d \sigma\left(e^{i l \sigma} S_{+}+e^{-i l \sigma} S_{-}\right) .
\end{aligned}
$$

Here $n$ are integers, while $l$ are integers or half-integers, corresponding to $\psi^{\mu}(\sigma)$ that satisfies the Ramond boundary condition $\psi_{+}^{\mu}(\pi)=\psi_{-}^{\mu}(\pi)$ (R sector) or the Neveu-Schwarz 
boundary condition $\psi_{+}^{\mu}(\pi)=-\psi_{-}^{\mu}(\pi)$ (NS sector), respectively. The global quantities $L_{n}$ and $F_{l}$ are referred to as super-Virasoro generators. To be more precise, these superVirasoro generators describe open strings. In the case of closed strings, there are two copies of super-Virasoro generators above, corresponding to right-moving and left-moving strings. Thus, for closed strings we have R-R, NS-NS, R-NS, and NS-R sectors.

In the quantum theory, the superstring coordinates become the operators $\hat{X}^{\mu}$ and $\hat{\psi}^{\mu}$. They satisfy the canonical equal-time (anti)commutation relations

$$
\begin{gathered}
{\left[\hat{X}^{\mu}(\sigma), \hat{P}^{\nu}\left(\sigma^{\prime}\right)\right]=-i \eta^{\mu \nu} \delta\left(\sigma-\sigma^{\prime}\right),} \\
\left\{\hat{\psi}_{b}^{\mu}(\sigma), \hat{\psi}_{b^{\prime}}^{\nu}\left(\sigma^{\prime}\right)\right\}=-\eta^{\mu \nu} \delta_{b b^{\prime}} \delta\left(\sigma-\sigma^{\prime}\right),
\end{gathered}
$$

where $b=1,2$ is the world-sheet spinor index and $\hat{P}^{\mu}=\partial \hat{X}^{\mu} / \partial \tau$. Thus, the superVirasoro generators (28) and (29) also become the operators, while the constraints (27) become the constraints on physical states

$$
\begin{gathered}
\hat{F}_{l}|\Psi\rangle=0, \quad l \geq 0, \\
\hat{L}_{n}|\Psi\rangle=0, \quad n>0, \\
\left(\hat{L}_{0}-a\right)|\Psi\rangle=0 .
\end{gathered}
$$

Here $a$ is a constant that depends on the ordering of the operators. When the normal ordering is used, then $a=1 / 2$ for the NS sector and $a=0$ for the R sector. It turns out that the quantum super-Virasoro constraints (32) are consistent only for $D=10$. The NS sector corresponds to bosonic states in spacetime, while the R sector corresponds to fermionic states in spacetime. However, not all states satisfying (32) are physical. The physical spectrum is a truncated one obtained from the spectrum above by the socalled GSO projection, which introduces further symmetry of the spectrum - spacetime supersymmetry [3, 4].

The correspondence between strings and particles can be seen by considering the modes with $n=l=0$. From (28) we see that $L_{0}$ is the Hamiltonian $\int d \sigma T_{00}$ with a term quadratic in the momenta $P^{\mu}$ (see also (33) below), so the last equation in (32) represents a stringy analog of the Klein-Gordon equation. In addition, the operator $\hat{F}_{0}$ satisfies $\hat{F}_{0} \hat{F}_{0}=\hat{L}_{0}$, so the equation $\hat{F}_{0}|\Psi\rangle=0$ of the fermionic $\mathrm{R}$ sector can be viewed as a stringy analog of the Dirac equation. Note, however, that a stringy analog of the Klein-Gordon equation is satisfied by both sectors, while that of the Dirac equation is satisfied only by one of the sectors. This fact will be crucial in construction of the unifying superstring current in the next subsection.

\subsection{Schrödinger picture and the superstring current}

In this subsection, we want to construct a superstring current that represents an analog of the particle current $j_{\mu}$ discussed in Sec. 2. A natural starting point is to write the last equation in (32) in a form that resembles the Klein-Gordon equation more explicitly, and similarly for the equation $\hat{F}_{0}|\Psi\rangle=0$ that resembles the Dirac equation. In particular, the momentum $\hat{P}_{\mu}$ should be represented by a derivative operator, analogously to the particle momentum operator $\hat{p}_{\mu}=i \partial_{\mu}$. For that purpose, we need to introduce the Schrödinger picture of quantum superstring theory. 
Before starting with the explicit construction, the following observations are crucial. In analogy with the standard approach to particles in Sec. 2.1, one could attempt to construct one superstring current for the bosonic NS sector and another superstring current for the fermionic R sector. However, according to the quantum superposition principle, a general quantum state is a superposition of states from both sectors. This fact does not have an analog at the level of (first-quantized) particle wave functions; a superposition of solutions of the Klein-Gordon and the Dirac equation does not make sense. This reflects the fact that in particle physics, as well as in quantum field theory, different particle species correspond to genuinely different objects. On the other hand, in superstring theory different particle species are only different states of the same object - the superstring. Therefore, in a consistent superstring theory there should be only one superstring current describing both NS and R sectors, as well as their arbitrary superpositions. Consequently, as both sectors satisfy the stringy analog of the Klein-Gordon equation, it is the stringy analog of the Klein-Gordon equation, and not that of the Dirac equation, from which the superstring current should be constructed.

Now we are ready for the explicit construction. From the action (22) one finds the Hamiltonian

$$
H=-\frac{1}{2} \int d \sigma\left[P^{\mu} P_{\mu}+\left(\partial_{\sigma} X^{\mu}\right)\left(\partial_{\sigma} X_{\mu}\right)-i \psi^{\top \mu} \rho^{0} \rho^{1} \partial_{\sigma} \psi_{\mu}\right]
$$

where $P^{\mu}$ and $\psi^{\mu}$ are functions of $\sigma, \partial_{\sigma} \equiv \partial / \partial \sigma$, and $\psi^{\dagger}=\psi^{\top}$ for a Majorana spinor. In the Schrödinger picture, the operator $\hat{P}_{\mu}(\sigma)$ is represented by the functional derivative

$$
\hat{P}_{\mu}(\sigma)=i \frac{\delta}{\delta X^{\mu}(\sigma)}
$$

which is consistent with (30). Various boundary conditions are imposed on the states $\Psi$, not on the operators. The explicit representation of the operator $\hat{\psi}^{\mu}(\sigma)$ will not be needed here, but we note that it is a direct sum of the corresponding operators for all sectors (such as open $\mathrm{R}$ sector, closed R-R sector, etc.). For our purposes, it is sufficient to know that $\hat{\psi}^{\mu}$ acts in some Hilbert space with indices $M$ and that the basis on this Hilbert space can be chosen such that a different index $M(\sigma)$ is attributed to each $\sigma$. Therefore, the last equation in (32) can be written in the Schrödinger picture as

$$
(\hat{H}-a) \Psi[X, M]=0,
$$

where $\Psi[X, M]$ is a functional of $X(\sigma)$ and $M(\sigma)$, while

$$
\hat{H}=\int d \sigma\left[\frac{\eta^{\mu \nu}}{2} \frac{\delta}{\delta X^{\mu}(\sigma)} \frac{\delta}{\delta X^{\nu}(\sigma)}-\frac{1}{2} \frac{\partial X^{\mu}(\sigma)}{\partial \sigma} \frac{\partial X_{\mu}(\sigma)}{\partial \sigma}+\hat{\mathcal{H}}_{\mathrm{F}}\right],
$$

with

$$
\hat{\mathcal{H}}_{\mathrm{F}} \equiv \frac{i}{2} \eta_{\mu \nu} \hat{\psi}^{\top \mu}(\sigma) \rho^{0} \rho^{1} \partial_{\sigma} \hat{\psi}^{\nu}(\sigma)
$$

The quantity $a$ can be viewed as a hermitian operator with different eigen-values on different sectors, making (35) correct even for an arbitrary superposition of states from different sectors. (Recall that the total Hilbert space is the direct sum of the Hilbert 
spaces of different sectors.) The fermionic part $\hat{\mathcal{H}}_{\mathrm{F}}$ contains the derivative $\partial_{\sigma}$, which shows that $\hat{\mathcal{H}}_{\mathrm{F}}$ vanishes in the pointlike-particle limit. Thus, in this limit, only the first term in (36) survives, leading to the Klein-Gordon equation.

Similarly, the supercurrent (25) becomes the operator

$$
\hat{S}_{\alpha}=\frac{1}{2} \rho^{0} \rho_{\alpha} \hat{\psi}^{\mu} \hat{P}_{\mu}+\frac{1}{2} \rho^{1} \rho_{\alpha} \hat{\psi}^{\mu} \partial_{\sigma} X_{\mu} .
$$

Again, the second term vanishes in the pointlike-particle limit owing to the derivative $\partial_{\sigma}$. The first term is proportional to $\hat{\psi}^{\mu} \hat{P}_{\mu}$, which looks similar to the Dirac operator $\gamma^{\mu} \hat{p}_{\mu}$. Indeed, comparing (31) with (5), we see that $\hat{\psi}^{\mu}$ are a sort of stringy analog of the Dirac matrices. More precisely, in $\mathrm{R}$ sector the relation is of the form [3]

$$
\gamma^{\mu} \propto \int d \sigma \hat{\psi}_{-}^{\mu}(\sigma)=\int d \sigma \hat{\psi}_{+}^{\mu}(\sigma) .
$$

Thus, it turns out that the pointlike-particle limit of the superstring constraint

$$
\hat{F}_{0} \Psi[X, M]=0
$$

is the Dirac equation. However, from (39) we see that, at the fundamental superstring level, $\gamma^{\mu}$ emerges from a Lorentz vector. Similarly, at the fundamental superstring level, the Dirac wave function $\varphi$ emerges from a Lorentz scalar $\Psi$. This confirms our assertion in Sec. 2.4 that, at the fundamental level, $\gamma^{\mu}$ corresponds to a vector and $\varphi$ to a collection of scalars.

To avoid confusion, we stress that $\Psi$ and $\varphi$ are scalars under spacetime coordinate transformations, but not under internal transformations in the Hilbert space with indices $M$. In particular, the spinor representation of the algebra of $\gamma^{\mu}$ 's corresponds to a tiny subspace of the whole Hilbert space in which $\hat{\psi}^{\mu}$ live. Thus, this internal spinor representation corresponds to the common description of fermions in curved spacetime, but does not play any fundamental role at the unifying superstring level.

Now, by analogy with the particle current studied in Sec. 2, the superstring current associated with the stringy analog of the Klein-Gordon equation, Eq. (35)), is

$$
J_{\mu}[X ; \sigma)=i \int[d M] \Psi^{*}[X, M] \frac{\overleftrightarrow{\delta}}{\delta X^{\mu}(\sigma)} \Psi[X, M] .
$$

Here the notation $J_{\mu}[X ; \sigma)$ denotes a functional with respect to $X(\sigma)$ and a function with respect to $\sigma$, while $[d M]$ denotes the functional integration over all $M(\sigma)$. We see that the superstring current (41) generalizes both (2) and (14). However, the fundamental superstring current (41) does not require a preferred foliation of spacetime, confirming the conjecture in Sec. 2.4 that the preferred foliation appears only at the level of effective field theory. In addition, the superstring current does not depend on the value of the parameter $a$ (this is analogous to the fact that the particle current does not depend on the mass $m$ ), which shows that the same current can be used for both R and NS sectors. Finally, since $\hat{\mathcal{H}}_{\mathrm{F}}$ in (36) is a hermitian operator that acts trivially in the space of functionals of $X$, and since the dependence on $M$ is integrated out in (41), the presence of the term $\hat{\mathcal{H}}_{\mathrm{F}}$ in (36) does not spoil the fact that the superstring current (41) is conserved, in the sense that

$$
\int d \sigma \frac{\delta}{\delta X^{\mu}(\sigma)} J^{\mu}[X ; \sigma)=0 .
$$


Note that $X$ and $M$ in (41) and (42) are not treated on an equal footing, which means that the world-sheet supersymmetry is not manifest. However, the world-sheet supersymmetry should be distinguished from the spacetime supersymmetry. The spacetime bosons and fermions are unified in the sense that the same current $J^{\mu}$ describes both spacetime bosons and spacetime fermions.

\section{The Bohmian interpretation}

\subsection{Bohmian interpretation of particles}

What is the physical interpretation of the current (2) associated to the wave function (19)? As the time component $j_{0}(x)$ may be negative, it cannot be interpreted as the probability density of particle positions. The time component cannot be interpreted as the charge density either, because the hermitian field $\hat{\phi}$ cannot describe a charged particle. A viable interpretation (see, e.g., [33, 34]) is the Bohmian interpretation, according to which the current determines the trajectory of the particle $X^{\mu}(\tau)$ according to the law

$$
\frac{d X^{\mu}}{d \tau}=j^{\mu}(X),
$$

where $\tau$ is an affine parameter along the trajectory. Thus, the trajectory is nothing but an integral curve of the vector field $j^{\mu}(x)$. As such, the trajectory does not depend on the parametrization with $\tau$. The reparametrization of all trajectories in the congruence of integral curves is equivalent to a local rescaling of the current

$$
j^{\mu}(x) \rightarrow j^{\prime \mu}(x)=e^{\Omega(x)} j^{\mu}(x)
$$

in Eq. (43), where $\Omega(x)$ is an arbitrary real function. If $j^{\mu}$ is conserved, i.e., if $\partial_{\mu} j^{\mu}=0$, then $j^{\prime \mu}$ may not be conserved:

$$
\partial_{\mu} j^{\prime \mu}=j^{\prime \mu} \partial_{\mu} \Omega .
$$

Thus, for the consistency of the Bohmian interpretation (43), the current $j^{\mu}$ does not necessarily need to be conserved.

By writing $\varphi=R e^{i S}$, where $R$ and $S$ are real functions, the current (2) is equal to $j_{\mu}=-2 R^{2} \partial_{\mu} S$. By a local rescaling this can be transformed to $j_{\mu}=-\partial_{\mu} S$. Therefore, the equation of motion (43) can be written in the Hamilton-Jacobi form as

$$
\frac{d X^{\mu}}{d \tau}=-\partial^{\mu} S(X) .
$$

However, the existence of such a Hamilton-Jacobi form is a consequence of the special form of the spin-0 current (2). For other spins, such a Hamilton-Jacobi form for the particle equation of motion does not exist. In particular, for the current (13) where $\varphi$ is a many-component wave function, a Hamilton-Jacobi form does not exist. Instead, for arbitrary spin, the motion of the particle is described by an equation of the form of (43) with an appropriate particle current $j^{\mu}$.

Now let us generalize this to the many-particle case. For simplicity, consider a manyparticle wave function $\varphi\left(x_{1}, \ldots, x_{n}\right)$ describing $n$ particles with spin 0 . Thus, there are $n$ 
particle currents $j_{a}^{\mu}=i \varphi^{*} \stackrel{\leftrightarrow}{\partial} \mu, a=1, \ldots, n$, generalizing the current (2). Each current is conserved, i.e., $\partial_{a \mu} j_{a}^{\mu}=0$ for each $a$. Consequently, there is also an overall conservation equation

$$
\sum_{a} \partial_{a \mu} j_{a}^{\mu}=0
$$

Therefore, the Bohmian equation of motion (43) generalizes to $n$ coupled equations [34]

$$
\frac{d X_{a}^{\mu}}{d \tau}=j_{a}^{\mu}\left(X_{1}, \ldots, X_{n}\right)
$$

for $n$ trajectories $X_{a}^{\mu}(\tau)$. For each $\tau$, the right-hand side of (48) depends on the positions of all particles at the same $\tau$. In other words, the velocity of one particle depends on the "instantaneous" position of all other particles. Such nonlocal "instantaneous" communication is exactly what is needed to make the nonlocal correlations typical of QM [11] consistent with the notion of objective reality. Nevertheless, we see that the equation of motion (48) is relativistic covariant, i.e., this equation does not depend on the choice of the preferred foliation. By a local rescaling, (48) can also be written in a form generalizing (46)

$$
\frac{d X_{a}^{\mu}}{d \tau}=-\partial_{a}^{\mu} S\left(X_{1}, \ldots, X_{n}\right)
$$

A remarkable property of the currents such as (2) and (13) is that these vector fields may be spacelike at some regions of spacetime, even when $\varphi$ is a superposition of positivefrequency solutions only. Consequently, the particle velocity (43) can exceed the velocity of light. As shown in [19], this does not contradict experiments because a measured velocity, associated to the eigenvalues of the velocity (or momentum) operator, can never exceed the velocity of light.

There are also qualitative suggestions that superluminal velocities with associated motions backwards in time may be related to the physical creation and destruction of particles. However, such superluminal motions appear even for free particles corresponding to the free Klein-Gordon equation (1), whereas the physical creation and destruction requires field interactions. Therefore, to make the existence of Bohmian particle trajectories consistent with the standard QFT predictions on particle creation and destruction, it turns out that one is forced to introduce some additional structure that, unfortunately, makes the theory less elegant. One possibility is to postulate stochastically chosen singular points at which particle trajectories begin or end, corresponding to particle creation or destruction [35, 36]. To avoid singular points and stochastic processes that break the spirit of the Bohmian interpretation, another possibility is to introduce an additional continuously evolving property of the particle called effectivity [19, 20], which leads to a picture in which particle trajectories never begin or end. Instead, the creation corresponds to a process in which the effectivity $e$ changes continuously from $e=0$ to $e=1$, while the destruction corresponds to a similar continuous fading from $e=1$ to $e=0$. Admittedly, both possibilities seem somewhat artificial. However, as we shall see in the next subsection, the Bohmian interpretation of strings offers a much more elegant picture of the processes of particle creation and destruction. Moreover, superluminal velocities and associated motions backwards in time that may seem undesirable at the particle level turn out to play an appealing role in the Bohmian description of string creation and destruction, thus reinforcing the viability of particle currents that may lead to superluminal 


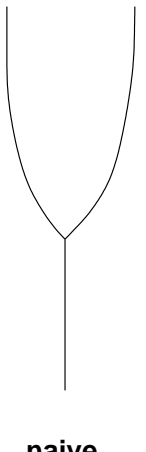

naive

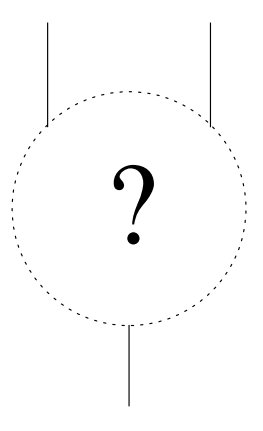

standard

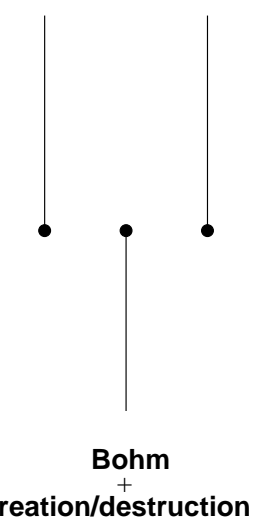

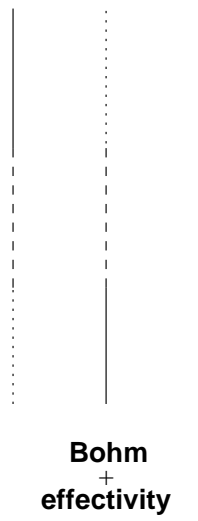

Figure 1: Various $1+1$ spacetime pictures of a decay of one particle into two particles. In a naive picture (which should not be confused with a similarly looking Feynman diagram), the particle literally splits into two particles, leading to a singular splitting point. No reasonable actual theory supports such a picture. In the standard S-matrix picture, only the measured asymptotic initial and final states are known, providing no answer to the question what happens at intermediate times at which measurements are not performed. In the Bohm+creation/destruction picture, particles suddenly and stochastically get created or destructed at singular points. In the Bohm+effectivity picture, particle trajectories never get created or destructed. Solid parts of trajectories correspond to fully effective particles with $e=1$, dashed parts correspond to $1>e>0$, while dotted parts correspond to ineffective ("virtual") particles with $e=0$. In the string-Bohm picture (which, again, should not be confused with a similarly looking Feynman diagram), the apparent string creation and destruction actually corresponds to a continuous distortion of a single string. The string boundary between the two crosses moves faster than light. Note that the most advanced string-Bohm picture is conceptually the most similar to the "naive" one, but contains no singular splitting point.

motions. Various pictures of particle creation and destruction, including the Bohmian string picture studied in the next section, are illustrated in Fig. 1.

\subsection{Bohmian interpretation of strings}

Let us start with the Bohmian interpretation of bosonic (rather than supersymmetric) string theory. In the bosonic case, the term $\hat{\mathcal{H}}_{\mathrm{F}}$ in (36) is absent. Consequently, there are no $M$-indices and the string wave functional is of the form $\Psi[X]=R[X] e^{i S[X]}$, where $R$ and $S$ are real. The Bohmian interpretation introduces a deterministic evolution of the string coordinates $X^{\mu}(\sigma, \tau)$, given by an equation of motion having a Hamilton-Jacobi form [15, 14]

$$
\frac{\partial X^{\mu}(\sigma, \tau)}{\partial \tau}=-\eta^{\mu \nu} \frac{\delta S[X]}{\delta X^{\nu}(\sigma)}
$$

which is analogous to (46). In (50), $\sigma$ and $\tau$ play different roles, which breaks the manifest world-sheet covariance of the Bohmian interpretation. However, there is a way to write (50) in a world-sheet covariant form [15], with a dynamically generated preferred foliation 
of the world-sheet (not of spacetime!). Another way to write (50) is

$$
\frac{\partial X^{\mu}(\sigma, \tau)}{\partial \tau}=\frac{1}{2 R^{2}[X]} J^{\mu}[X ; \sigma),
$$

where

$$
J_{\mu}[X ; \sigma)=i \Psi^{*}[X] \frac{\overleftrightarrow{\delta}}{\delta X^{\mu}(\sigma)} \Psi[X]
$$

Analogously to that in Sec. 4.1, the parameter $\tau$ can be redefined so that the factor $1 / 2 R^{2}$ in (51) gets eliminated, leading to the equation analogous to (43)

$$
\frac{\partial X^{\mu}(\sigma, \tau)}{\partial \tau}=J^{\mu}[X ; \sigma)
$$

At this point it is fair to remark that the string Bohmian equations of motion different from (50) are also conceivable. Nevertheless, there are several theoretical arguments for preferring (50). First, (50) is the obvious generalization of the particle Bohmian equation of motion (46). Second, (50) represents the obvious generalization of classical string dynamics, as the classical Hamilton-Jacobi string equation of motion takes the same form as (50) [15]. Third, the equation of motion (50) can be derived from the worldsheet covariant canonical quantization of strings introduced in [15], where the Bohmian equation of motion is derived (not simply postulated for interpretational purposes) from the requirement that the world-sheet covariant canonical quantization based on the De Donder-Weyl formalism should be compatible with the standard canonical quantization. Fourth, (50) has a property of manifest spacetime relativistic covariance. Some additional arguments for preferring (50) will be presented in Sec. 5 .

In [15, 14], the Bohmian interpretation was studied for free bosonic strings only. However, one of the most remarkable properties of perturbative string theory (i.e., theory defined by the perturbative expansion over Feynman diagrams with different world-sheet topologies) is the fact that the string analog of particle creation and destruction is also described by the free string Lagrangian. (The specific interactions of the low-energy fieldtheoretic 4-dimensional Standard Model are encoded in a peculiar relation between the observed 4 dimensions and the unobserved extra dimensions of string theory.) Thus, the free string wave functional $\Psi[X]$ also contains the information about the probabilities for string creation and destruction. To see how, the crucial observation is that the functional $\Psi[X]$ may be nonvanishing even for functions $X^{\mu}(\sigma)$ that are not continuous. For example, a discontinuous function of the form

$$
X^{\mu}(\sigma)=\left\{\begin{array}{l}
X_{1}^{\mu}(\sigma) \text { for } 0 \leq \sigma<\frac{\pi}{2} \\
X_{2}^{\mu}(\sigma) \text { for } \frac{\pi}{2}<\sigma \leq \pi
\end{array}\right.
$$

where $X_{1}^{\mu}(\sigma)$ and $X_{2}^{\mu}(\sigma)$ are continuous functions, may be viewed as a configuration that describes two strings. Consequently, for such functions, one can write $\Psi[X]=\Psi\left[X_{1}, X_{2}\right]$, describing a state with two strings. For entangled strings corresponding to the case $\Psi[X]=\Psi\left[X_{1}, X_{2}\right] \neq \Psi_{1}\left[X_{1}\right] \Psi_{2}\left[X_{2}\right]$, the Bohmian interpretation requires a preferred foliation of the world-sheet (which can be introduced dynamically [15]), but a preferred 
foliation of spacetime is not needed here. Nevertheless, a relation with the foliation of spacetime exists: the vector $n^{\mu}(x)$ associated with the foliation of spacetime can be projected onto the world-sheet, which then defines a foliation of the world-sheet as well. This does not uncover a more fundamental origin of the preferred foliation, but suggests that Bohmian nonlocality and string theory may be related in a yet undiscovered way. (For an overview of nonlocal phenomena emerging from string theory, see [12, 13], and for a more stringent relation with Bohmian nonlocalities, see [14].)

To see more explicitly how the description of string creation and destruction in the Schrödinger picture is related to the usual formulation of string theory [3, 4], we proceed as follows. We start from the functional Schrödinger equation

$$
\hat{H} \Psi[X ; \tau)=i \partial_{\tau} \Psi[X ; \tau),
$$

where, for convenience, the constant $a$ is absorbed into a redefinition of the Hamiltonian $\hat{H}$. As is well known from particle quantum mechanics and QFT [31], the evolution described by the Schrödinger equation is equivalent to

$$
\Psi[X ; \tau)=\int\left[d X^{\prime}\right]\left\langle X ; \tau \mid X^{\prime} ; \tau_{0}\right\rangle \Psi\left[X^{\prime} ; \tau_{0}\right),
$$

where $[d X]$ is a functional integral with respect to all $X^{\mu}(\sigma)$, the propagator $\left\langle X ; \tau \mid X^{\prime} ; \tau_{0}\right\rangle$ is given by the path integral

$$
\left\langle X_{A} ; \tau_{A} \mid X_{B} ; \tau_{B}\right\rangle=\int_{X_{A}(\sigma), \tau_{A}}^{X_{B}(\sigma), \tau_{B}}[d X(\sigma, \tau)] e^{i A[X]},
$$

and $A$ is the classical action (22) (without the fermionic part depending on $\psi^{\mu}$, as we deal here with bosonic string theory). In the path integral above, various functions $X^{\mu}(\sigma, \tau)$ may be discontinuous at various points and can be classified according to different topologies of the corresponding world-sheets. Therefore, (57) can be further written as

$$
\left\langle X_{A} ; \tau_{A} \mid X_{B} ; \tau_{B}\right\rangle=\sum_{g} \int_{X_{A}(\sigma), \tau_{A}}^{X_{B}(\sigma), \tau_{B}}[d X(\sigma, \tau)]_{g} e^{i A[X]},
$$

where $[d X(\sigma, \tau)]_{g}$ denotes the integration over functions that describe the same topology. So far we have been assuming that $\Psi$ satisfies the Schrödinger equation (55), but actually this is not the only equation that $\Psi$ satisfies. First, it also satisfies the Hamiltonian constraint $\hat{H} \Psi=0$, which means that $\Psi$ in (55) does not really depend on $\tau$, and consequently, that the propagators (57) and (58) do not really depend on $\tau_{A}$ and $\tau_{B}$. Second, it also satisfies all other Virasoro constraints for $n>0$ (see Eq. (32)), which implies that the propagator does not depend on the choice of the world-sheet metric $h_{\alpha \beta}(\sigma, \tau)$. Therefore, in (58), one must replace the implicit Minkowski world-sheet metric $\eta_{\alpha \beta}$ in (22) by an arbitrary metric $h_{\alpha \beta}(\sigma, \tau)$ and integrate over all possible metrics. This leads to

$$
\left\langle X_{A} \mid X_{B}\right\rangle=\sum_{g} \int_{X_{A}(\sigma)}^{X_{B}(\sigma)}[d h(\sigma, \tau)]_{g}[d X(\sigma, \tau)]_{g} e^{i A[X, h]},
$$

where $[d h(\sigma, \tau)]_{g}$ denotes the functional integral over all possible world-sheet metrics $h_{\alpha \beta}(\sigma, \tau)$ consistent with given topology of the world-sheet. (In the bosonic case, (59) turns out to be well defined only for $D=26$.) Consequently, (56) is replaced by

$$
\Psi[X]=\int\left[d X^{\prime}\right]\left\langle X \mid X^{\prime}\right\rangle \Psi\left[X^{\prime}\right]
$$


Eq. (59) is nothing but the usual path-integral formula for calculating the string scattering amplitudes, where the summation over $g$ corresponds to the summation over Feynman diagrams with different topologies. The quantity $\Psi[X]$ in (60) is the string wave functional that defines the bosonic string current (152).

As is well known from the Bohmian interpretation of particles and fields, solutions of the Bohmian equation of motion (50) can only attain configurations for which the amplitude of $\Psi[X]$ does not vanish. Moreover, when the quantum-mechanical probabilities are well-defined by $\Psi[X]$, then the corresponding probabilities predicted by the Bohmian interpretation turn out to be exactly the same. (We discuss it in more detail in Sec. 5.) Now, since $\Psi[X]$ contains amplitudes corresponding to different world-sheet topologies, including those that correspond to string splitting, it is evident that the Bohmian equation of motion (53) contains solutions corresponding to the same world-sheet topologies. In particular, some solutions describe deterministic processes of string splitting, which correspond to particle creation and destruction (see Fig. 1 for an example). In fact, such splitting solutions exist even in classical string theory [37]. In this way, unlike the Bohmian interpretation of pointlike particles, the Bohmian interpretation of strings does not need an artificial introduction of stochastic singular points at which the particle trajectories begin and end, or an artificial introduction of effectivities. Thus, the Bohmian interpretation of strings provides a very elegant solution to the problem of the Bohmian description of particle creation and destruction (see also [38]).

Now we also see why it is appealing that the current such as (52) may be locally spacelike. In the Bohmian interpretation this corresponds to local superluminal velocities, which, as demonstrated by Fig. 1, is related to string creation and destruction without leading to singular splitting points, provided that $S[X]$ is sufficiently smooth, leading to a smooth right-hand side of (50). (Without superluminal velocities, the U-shape of the string boundary between the two crosses would be replaced by a V-shape containing a singular splitting point at the cusp.)

Now the generalization of the bosonic-string results above to superstrings is straightforward, as we now discuss briefly. It is evident that the superstring generalization of (52) is the superstring current (41). Thus, the natural interpretation of this superstring current is the Bohmian interpretation defined by (53), i.e.,

$$
\frac{\partial X^{\mu}(\sigma, \tau)}{\partial \tau}=J^{\mu}[X ; \sigma)
$$

Note that, in the superstring case, there is no Bohmian equation of motion for the "classical" Grassmann valued coordinate $\psi^{\mu}$ in (22). This is analogous to the fact that in particle Bohmian mechanics there is no Bohmian equation of motion for the spin of the particle [39]. The spin degrees of freedom are integrated out in the particle case (14) in the same manner as the degrees of freedom attributed to $\hat{\psi}^{\mu}$ are integrated out in the superstring case (41). In this way, the Bohmian interpretation of superstrings provides a sort of explicit realization of the idea that in Bohmian mechanics "all particles are identical" [40, i.e., that all particles are merely different states of the same particle (which here turns out to be an extended "particle", that is - string). 


\section{Probabilistic predictions and consistency with the Bohmian interpretation}

In the previous section we have presented the deterministic Bohmian equations of motion for particles and strings. However, we have not explicitly explained how the usual probabilistic predictions of the conventional formulation of quantum theory can be recovered from these deterministic equations of motion. This is what we do in this section.

Our approach is based on the old idea [41, 42, 43, 44] that in relativistic QM the quantity $|\varphi(x)|^{2}$ represents the probability density of the particle position in spacetime, rather than that in space. Such an approach has an advantage that the probabilistic interpretation is manifestly relativistic covariant and can be easily generalized to many particles and strings, without introducing any preferred foliation of spacetime. Here, partially inspired by the results of [33], we construct a Bohmian version of that interpretation. Actually, we further refine the ideas of [33] by showing that, even with the Bohmian interpretation, the probabilistic predictions of particle positions are easily found on any hypersurface. (For further developments see also [45, 46].)

The simplest way to understand our approach is to exploit the analogy with the wellunderstood case of free nonrelativistic particles in an energy eigenstate, by discussing the latter from a somewhat unusual point of view. Thus, the first part of this subsection is devoted to a detailed discussion of the nonrelativistic case, while the most interesting relativistic case is then presented in the second part as a simple generalization.

\subsection{Nonrelativistic particles in an energy eigenstate}

A free nonrelativistic particle in an energy eigenstate is described by a wave function of the form $\psi(t, \mathbf{x})=e^{-i E t} \varphi(\mathbf{x})$, where $\mathbf{x} \equiv\left\{x^{1}, x^{2}, x^{3}\right\}$. Thus, the nonrelativistic Schrödinger equation reduces to the time-independent Schrödinger equation $-\nabla^{2} \varphi / 2 m=E \varphi$, which we write as

$$
\left(\nabla^{2}+M^{2}\right) \varphi(\mathbf{x})=0,
$$

where $M^{2} \equiv 2 m E$. We write the time-independent Schrödinger equation in the form (62) to make the analogy with the relativistic Klein-Gordon equation (1) obvious. The current

$$
\mathbf{j}=\frac{i \varphi^{*} \stackrel{\leftrightarrow}{\nabla} \varphi}{2 m}
$$

satisfies the space-conservation equation

$$
\nabla \mathbf{j}=0
$$

which are (up to an inessential normalization factor $1 / 2 m$ ) nonrelativistic analogs of (2) and (3) , respectively. How to interpret (64) in terms of conserved probabilities? Since $\varphi$ does not depend on $t$, (64) also trivially implies a conservation equation involving time

$$
\frac{\partial|\varphi|^{2}}{\partial t}+\nabla\left(|\varphi|^{2} \mathbf{v}\right)=0
$$


where $\mathbf{v} \equiv \mathbf{j} /|\varphi|^{2}=\nabla S / m$ and $\varphi=R e^{i S}$. Thus, it is consistent to postulate that the $a$ priori probability density of particle positions is

$$
p(\mathbf{x})=|\varphi(\mathbf{x})|^{2} .
$$

Consequently, it is also consistent to postulate that particles have trajectories $\mathbf{X}(\mathrm{t})$ satisfying

$$
\frac{d \mathbf{X}}{d t}=\frac{\nabla S}{m},
$$

which is a nonrelativistic analog of (46) (up to an additional inessential rescaling of $\tau$ in (46)). Indeed, (67) is the standard nonrelativistic Bohmian equation of motion.

In practice, one rarely measures the probability density (66) in the whole space. Instead, one frequently deals with a beam moving in the $x^{3}$ direction and measures particle positions on the detection screen at $x^{3}=z$. It is convenient to model the beam by a wave function localized in the $x^{1}-x^{2}$ directions, but infinitely extended in the $x^{3}$ direction. For example, the wave function may have the form

$$
\varphi(\mathbf{x})=e^{i k^{3} x^{3}} \chi\left(x^{1}, x^{2}\right),
$$

where $\chi\left(x^{1}, x^{2}\right)$ is a localized wave packet, but a more general dependence on $x^{3}$ is also possible. Thus we have

$$
\int_{-\infty}^{\infty} d x^{3}\left|\varphi\left(x^{1}, x^{2}, x^{3}\right)\right|^{2}=\infty .
$$

Nevertheless, the property (69) does not represent a practical problem, because the measured probability density of particle positions at $x^{3}=z$ is

$$
p_{z}\left(x^{1}, x^{2}\right)=\frac{\left|\varphi\left(x^{1}, x^{2}, z\right)\right|^{2}}{N_{z}},
$$

where

$$
N_{z}=\int d x^{1} d x^{2}\left|\varphi\left(x^{1}, x^{2}, z\right)\right|^{2}
$$

is the normalization factor. Even though the wave function cannot be normalized in the whole space due to (69), the physically relevant normalization factor (171) is finite. The probability density (70) is nothing but the conditional probability density derived from the joint probability density (66).

It is well known that (70) is consistent with the Bohmian interpretation, but it is crucial to understand why exactly this is so. The Bohmian trajectories are given by 3 functions

$$
X^{1}(t), X^{2}(t), X^{3}(t)
$$

satisfying (67). Now assume that an experimentalist is not equipped with a clock that measures $t$. In particular, he does not measure the time at which the particle approaches the detection screen at $x^{3}=z$. Thus, for practical purposes, the parameter $t$ can be thought of as an auxiliary mathematical parameter without a direct physical interpretation. Hence, it is convenient to eliminate $t$ from (72). From the last function in (72) one can find $t\left(X^{3}\right) \equiv t\left(x^{3}\right)$ and insert this into the first two functions. In this way one ends up with only two functions

$$
X^{1}\left(x^{3}\right), X^{2}\left(x^{3}\right) .
$$


Geometrically, (72) is a curve in $3+1=4$ dimensions, while $(73)$ is a curve in only $2+1=3$ dimensions. In fact, and this is the crucial point, the physical coordinate $x^{3}$ in (73) plays a role of a time coordinate. In this picture, $\psi\left(x^{1}, x^{2}, x^{3}\right)$ describes how the wave function evolves with "time" $x^{3}$, while (70) is the probability density on a surface of constant "time" $x^{3}=z$. The crucial point to note is that, with the dependence on $x^{3}$ more general than that in (68), the norm (71) depends on $z$. Consequently, the probability density on a surface of constant "time" is not simply given by the numerator on the right-hand side of (70). Nevertheless, the probability density is proportional to this numerator, with the proportionality factor being $z$-dependent as expressed by the denominator on the right-hand side of (70).

How can (70) be understood from the Bohmian $2+1$ point of view? In general, the trajectories (73) may have a very strange appearance from the $2+1$ perspective. For example, by allowing a more complicated dependence on $x^{3}$ in (68), the trajectories may even involve motions backwards in "time". Nevertheless, (170) is the correct probability density on $x^{3}=z$. This is because the a priori probability density on the whole space is given by (66). From the $2+1$ perspective, it means that not only the initial particle position $X^{1}\left(x_{\text {initial }}^{3}\right), X^{2}\left(x_{\text {initial }}^{3}\right)$ is unknown and hence subject to the probabilistic law, but also the value of the initial "time" $x_{\text {initial }}^{3}$ itself is unknown and hence subject to the probabilistic law.

Now let us briefly generalize all this to an $n$-particle energy eigenstate. Most of it is obvious, so let us only discuss some less obvious features. The $n$-particle energy eigenstate is described by a wave function $\varphi\left(\mathbf{x}_{1}, \ldots, \mathbf{x}_{n}\right)$. The a priori probability density (66) generalizes to

$$
\left.p\left(\mathbf{x}_{1}, \ldots, \mathbf{x}_{n}\right)=\mid \varphi\left(\mathbf{x}_{1}, \ldots, \mathbf{x}_{n}\right)\right)\left.\right|^{2} .
$$

Since it does not depend on $t$, it is the correct joint probability density even if different particles are detected at different $t$. (Note that the calculation of the joint probability density does not involve an effective collapse of the wave function. The collapse is only related to the calculation of a conditional probability density, such as calculating the probability that the particle 2 has the position $\mathbf{x}_{2}$ at time $t_{2}$, given that the particle 1 has been found at the position $\mathbf{x}_{1}$ at time $t_{1}$.) As in the 1-particle case above, we are interested in a situation in which $t$ is an unmeasured (and hence effectively unphysical) parameter. In general, it can be arranged that different particles are detected on screens positioned at different values of $x^{3}$. Hence, (70)-(71) generalize to

$$
\begin{gathered}
p_{z_{1}, \ldots, z_{n}}\left(\mathbf{x}_{1}^{\perp}, \ldots, \mathbf{x}_{n}^{\perp}\right)=\frac{\left|\varphi\left(\mathbf{x}_{1}^{\perp}, z_{1}, \ldots, \mathbf{x}_{n}^{\perp}, z_{n}\right)\right|^{2}}{N_{z_{1}, \ldots, z_{n}}} \\
N_{z_{1}, \ldots, z_{n}}=\int d^{2} \mathbf{x}_{1}^{\perp} \ldots \int d^{2} \mathbf{x}_{n}^{\perp}\left|\varphi\left(\mathbf{x}_{1}^{\perp}, z_{1}, \ldots, \mathbf{x}_{n}^{\perp}, z_{n}\right)\right|^{2},
\end{gathered}
$$

where $\mathbf{x}_{a}^{\perp} \equiv\left\{x_{a}^{1}, x_{a}^{2}\right\}$. The Bohmian trajectories $\mathbf{X}_{a}(t)$ can be viewed as $n$ curves in 4 dimensions. But time can be eliminated, leading to functions $\mathbf{X}_{a}^{\perp}\left(x_{a}^{3}\right)$, which can be viewed as $n$ curves in 3 dimensions $\mathbf{X}_{a}^{\perp}\left(x^{3}\right)$. Now (75) is consistent with the trajectories $\mathbf{X}_{a}^{\perp}\left(x^{3}\right)$ because not only the initial conditions $\mathbf{X}_{a}^{\perp}\left(x_{a}^{3}\right.$ initial $)$ are subject to the probabilistic law (74), but so are the initial "times" $x_{a \text { initial }}^{3}$ themselves. 


\subsection{Relativistic particles and strings}

Now we are finally ready to deal with the relativistic case. Actually, with the nonrelativistic case being described as above, the generalization to the relativistic case is very simple. Essentially, all we need to do is to add one dimension more. More precisely, the transition from a nonrelativistic particle in an energy eigenstate to a relativistic particle is accomplished through the following correspondence:

$$
\begin{gathered}
t \rightarrow \tau, \\
\mathbf{x}=\left(x^{1}, x^{2}, x^{3}\right) \rightarrow x=\left(x^{0}, x^{1}, x^{2}, x^{3}\right), \\
\mathbf{x}^{\perp}=\left(x^{1}, x^{2}\right) \rightarrow \mathbf{x}=\left(x^{1}, x^{2}, x^{3}\right), \quad x^{3} \rightarrow x^{0} .
\end{gathered}
$$

In particular, the parameter $\tau$ in (46) can be thought of as a scalar time corresponding to a fifth dimension [41, 42, 43, 44]. However, $\tau$ is an auxiliary unphysical parameter, i.e., we are not equipped with a clock that measures it. Hence it is natural to eliminate $\tau$, so that (46) describes a curve in 4 physical dimensions. The wave function $\varphi(x)$ does not depend on $\tau$, so (65) generalizes to

$$
\frac{\partial|\varphi|^{2}}{\partial \tau}+\partial_{\mu}\left(|\varphi|^{2} v^{\mu}\right)=0
$$

where $v^{\mu} \equiv j^{\mu} /|\varphi|^{2}=-\partial^{\mu} S$ and the second term in (78) vanishes due to (3). Consequently, (66) generalizes to

$$
p(x)=|\varphi(x)|^{2},
$$

which defines a probability density conserved in $\tau$. Since $\tau$ is unphysical, (79) can also be thought of as the a priori probability density that particle will be found at any point $x$ in spacetime. In other words, (79) is the probability density of flashes in spacetime.

At this point it is interesting to note that flashes in spacetime have also been introduced in a different context [47], as a way to make the objective-collapse interpretation of QM fully compatible with relativity. In our approach, (79) will be the ultimate reason why the probabilistic predictions of the Bohmian interpretation are fully compatible with relativity. Thus, in both cases the ultimate origin of the compatibility between relativity and hidden variables for QM lies in a use of flashes in spacetime, which cannot be a coincidence. Nevertheless, in our Bohmian approach the particles are not flashes but continuous trajectories in spacetime, while the flashes correspond only to initial conditions. Namely, a Bohmian trajectory can be specified by fixing $X^{\mu}(\tau=0)$, which, after elimination of the variable $\tau$, means that one must fix $X^{i}\left(x_{\text {initial }}^{0}\right), i=1,2,3$, as well as $x_{\text {initial }}^{0}$ itself. The a priori probability density for such an initial condition is given by (79).

The probability density (79) cannot be normalized in the whole spacetime, due to an analog of (69)

$$
\int d x^{0}\left|\varphi\left(x^{0}, x^{1}, x^{2}, x^{3}\right)\right|^{2}=\infty .
$$

Nevertheless, this is not a problem in practice if one is interested in the probability for a fixed $x^{0}$. If one is certain that a particle will be detected at the time $x^{0}=t$, then the probability distribution of particle positions in space at $x^{0}=t$ is given by the conditional probability density

$$
p_{t}(\mathbf{x})=\frac{|\varphi(t, \mathbf{x})|^{2}}{N_{t}},
$$


where

$$
N_{t}=\int d^{3} x|\varphi(t, \mathbf{x})|^{2} .
$$

These equations are nothing but obvious generalizations of (70) and (71). In nonrelativistic QM, where $\varphi(t, \mathbf{x})$ satisfies a first-order differential equation with respect to $t$, the norm (82) does not depend on $t$. In relativistic QM described by the Klein-Gordon equation this norm may depend on $t$ (actually, it depends on $t$ only if $\varphi$ is a superposition of solutions with both positive and negative frequencies [48]), which is the standard argument that $|\varphi|^{2}$ is not the probability density at fixed time in relativistic QM. Nevertheless, now we see that the probability density at fixed time is proportional to $|\varphi|^{2}$, with the time-dependent proportionality factor given by the denominator on the right-hand side of $(81)$.

Given the a priori probability density of flashes (79), it is clear that (81) is the correct conditional probability density. However, in nonrelativistic QM, (81) (with constant $N_{t}$ ) is interpreted as an a priori probability density, i.e., one allways assumes that one is certain that the particle will be detected at any fixed value of $t$. Can one be certain that this will also be the case when one starts from (79)? While it may be a difficult conceptual question within the orthodox probabilistic interpretation of QM (which may be a reason why the probabilistic interpretation of QM advocated in [41, 42, 43, 44] is not widely accepted), this question has a simple answer within the Bohmian interpretation. Since particles are actually trajectories (not merely flashes) in spacetime, one expects that every trajectory associated with a free Klein-Gordon equation crosses any given hypersurface of equal time. Indeed, we have performed some numerical computations that give evidence that this is indeed the case when $\varphi$ is a superposition of positive-frequency solutions only. Consequently, one can be certain that a particle will be detected at any fixed value of $t$ (assuming, of course, an ideal detector with perfect efficiency).

Now we can generalize it to $n$-particle relativistic QM. Due to (47), the conservation equation (78) generalizes to

$$
\frac{\partial|\varphi|^{2}}{\partial \tau}+\sum_{a} \partial_{a \mu}\left(|\varphi|^{2} v_{a}^{\mu}\right)=0
$$

where $v_{a}^{\mu}$ is the right-hand side of (49). Consequently, by analogy with (74), (79) generalizes to

$$
p\left(x_{1}, \ldots, x_{n}\right)=\left|\varphi\left(x_{1}, \ldots, x_{n}\right)\right|^{2},
$$

which is consistent with the Bohmian equation of motion (49). Similarly, by analogy with (75)-(76), (81)-(82) generalize to

$$
\begin{gathered}
p_{t_{1}, \ldots, t_{n}}\left(\mathbf{x}_{1}, \ldots, \mathbf{x}_{n}\right)=\frac{\left|\varphi\left(t_{1}, \mathbf{x}_{1}, \ldots, t_{n}, \mathbf{x}_{n}\right)\right|^{2}}{N_{t_{1}, \ldots, t_{n}}} \\
N_{t_{1}, \ldots, t_{n}}=\int d^{3} x_{1} \cdots \int d^{3} x_{n}\left|\varphi\left(t_{1}, \mathbf{x}_{1}, \ldots, t_{n}, \mathbf{x}_{n}\right)\right|^{2} .
\end{gathered}
$$

Eq. (85) is nothing but the conditional probability density associated with the a priori joint probability density (84). It can also be generalized to the case in which the hypersurfaces of constant $x_{a}^{0}$ are replaced by arbitrary curved spacelike hypersurfaces $\Sigma_{a}$. 
On such general hypersurfaces one needs to know the determinants of the induced metric $g_{a}^{(3)}\left(\mathbf{x}_{a}\right)$, where $\mathbf{x}_{a}$ are the coordinates on $\Sigma_{a}$. Eqs. (85)- (86) then further generalize to

$$
\begin{gathered}
\tilde{p}_{\Sigma_{1}, \ldots, \Sigma_{n}}\left(\mathbf{x}_{1}, \ldots, \mathbf{x}_{n}\right)=\frac{\sqrt{\left|g_{1}^{(3)}\left(\mathbf{x}_{1}\right)\right|} \cdots \sqrt{\left|g_{n}^{(3)}\left(\mathbf{x}_{n}\right)\right|}\left|\varphi\left(x_{1}, \ldots, x_{n}\right)\right|^{2}}{N_{\Sigma_{1}, \ldots, \Sigma_{n}}}, \\
N_{\Sigma_{1}, \ldots, \Sigma_{n}}=\int_{\Sigma_{1}} d^{3} x_{1} \sqrt{\left|g_{1}^{(3)}\left(\mathbf{x}_{1}\right)\right|} \cdots \int_{\Sigma_{n}} d^{3} x_{n} \sqrt{\left|g_{n}^{(3)}\left(\mathbf{x}_{n}\right)\right|}\left|\varphi\left(x_{1}, \ldots, x_{n}\right)\right|^{2} .
\end{gathered}
$$

The tilde on $\tilde{p}$ denotes that it transforms as a scalar density, which in curved coordinates should be distinguished from a quantity that transforms as a scalar.

What remains is to generalize all this to bosonic strings and superstrings. The generalization to bosonic strings is trivial because all one needs to do is to replace the discrete label $a$ by a continuous variable $\sigma$. In particular, the analog of (84) is

$$
p[X]=|\Psi[X]|^{2},
$$

and similarly for the other equations. For superstrings, it further generalizes to

$$
p[X]=\int[d M]|\Psi[M, X]|^{2} .
$$

Indeed, $\tau$ in (61) can be rescaled, such that the Bohmian equation of motion becomes

$$
\frac{\partial X^{\mu}(\sigma, \tau)}{\partial \tau}=\frac{J^{\mu}[X ; \sigma)}{p[X]} .
$$

This is consistent with the conservation equation

$$
\frac{\partial p}{\partial \tau}+\int d \sigma \frac{\delta}{\delta X^{\mu}(\sigma)} J^{\mu}[X ; \sigma)=0
$$

that results from (42).

Few additional remarks are in order. First, the probabilistic interpretation (89) requires that $\Psi[X]$ should be formally normalized such that $\int[d X]|\Psi[X]|^{2}=1$. However, the integral $\int[d X]|\Psi[X]|^{2}$ is actually infinite, so such a normalization does not exist in a strict sense. Nevertheless, this integral can be made finite by replacing the measure $[d X]$ by an appropriately regularized measure. (For example, $\sigma$ can be replaced by a discrete variable and the range of values of $X^{\mu}$ can be taken to be finite.) At the end of calculation the regularization parameters can be turned back to their natural values. One does not expect that such manipulations would affect the final measurable results, but a detailed analysis of these technical subtleties is beyond the scope of the present paper.

Second, the probabilistic interpretation (89) or (190) is not the probabilistic interpretation used in standard applications of string theory. In fact, in standard applications of string theory one does not discuss a probabilistic interpretation in the configuration space at all. Instead, one is usually interested in probabilities that the string will end up in a state $\Psi_{A}[X, M]$, where $\Psi_{A}[X, M]$ is a state that has definite values of particle-like properties (such as energy, momentum, and spin) interesting in scattering experiments. By writing

$$
\Psi[X, M]=\sum_{A} c_{A} \Psi_{A}[X, M]
$$


the appropriate probabilities are $p_{A}=\left|c_{A}\right|^{2}$. The standard applications of string theory are concentrated on practical methods for calculation of the amplitudes $c_{A}$. However, a complete quantum description must also involve a description of the measuring apparatus. Thus, instead of (93), we actually have the total wave functional

$$
\Psi_{\text {total }}\left[X_{\text {total }}, M_{\text {total }}\right]=\sum_{A} c_{A} \Psi_{A}[X, M] \Phi_{A}\left[X_{\text {app }}, M_{\text {app }}\right],
$$

where $\Phi_{A}\left[X_{\mathrm{app}}, M_{\mathrm{app}}\right]$ are normalized states of the measuring apparatus that do not over-

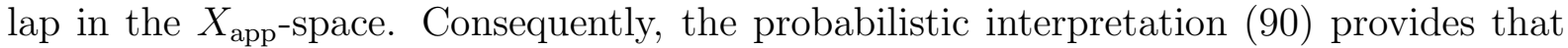
the probability that $X_{\text {app }}$ will take a value from the support of $\Phi_{A}\left[X_{\text {app }}, M_{\text {app }}\right]$ is equal to $\left|c_{A}\right|^{2}$. This shows that the probabilistic interpretation (89) is compatible with the standard one.

Third, the probabilistic interpretation (90) allows one additional argument for the equation of motion (91). Even without the Bohmian interpretation, the average local momentum of the string is

$$
\left\langle P^{\mu}(\sigma)\right\rangle=\int[d X] \int[d M] \Psi^{*}[X, M] \hat{P}^{\mu}(\sigma) \Psi[X, M]=\int[d X] \frac{J^{\mu}[X ; \sigma)}{2},
$$

where (34) and (41) have been used to obtain the second equality. We require that the average momentum of Bohmian trajectories should be equal to the quantum-mechanical average momentum (95). Since the Bohmian velocities have the same directions in spacetime as Bohmian momenta, the simplest way to accomplish this requirement is to postulate that the Bohmian velocities have the same directions as $J^{\mu}$. This leads to (61) equivalent to (91). Alternatively, instead of invoking the argument of simplicity, the same result can be obtained by applying a relativistic generalization of the argument in [50, 51] based on weak measurements of velocities. Of course, analogue arguments can also be applied to bosonic string theory without the $M$ variable.

Fourth, even though the Bohmian velocities (91) may be spacelike and past-oriented in some cases, no deviations from predictions of standard quantum theory are possible when these velocities are measured, i.e., when the total state is of the form of (94) with $\Psi_{A}$ being the eigenstates of $\hat{P}^{\mu}$. In particular, superstring theory does not contain tachyons, so measured velocities cannot be spacelike.

\section{Conclusion}

This paper contains several new results on boson-fermion unification, string theory, and Bohmian mechanics. As our main aim is to emphasize the new conceptual aspects, some results can certainly be formulated more rigorously in the future. But what exactly are the problems that these results are supposed to solve? It depends on what one considers to be a problem.

From the perspective of a traditional theoretician not interested in foundations of quantum mechanics (QM), we have contributed to the understanding of boson-fermion unification by constructing unifying particle and superstring currents. We have also seen that these currents lead to a preferred spacetime foliation in effective field theory and indicated how it reinterprets the notion of particles in QFT in curved spacetime. Still, 
to fully understand the physical meaning of these currents, it is necessary to deal with foundations of QM itself.

From the perspective of physicists interested in various interpretations of QM, we have presented a new argument for the naturalness of the Bohmian interpretation, by observing that this is a natural interpretation of the unifying particle and superstring currents. In addition, we have further developed a relativistic covariant probabilistic interpretation of wave functions based on earlier ideas [41, 42, 43, 44].

From the perspective of physicists interested in the internal problems of the Bohmian interpretation, we have offered solutions to several fundamental problems of that interpretation. These problems and solutions may be summarized by the following items:

1. Fundamental ontology - only strings (appearing as particles at low energies) have an objective existence, for both fermions and bosons.

2. Creation and destruction of particles - corresponds to continuous string splitting described by the Bohmian equation of motion.

3. Superluminal Bohmian velocities - play an appealing role for string splitting without singular splitting points.

4. Preferred foliation of spacetime - appears to be related (in a surprising way) to the boson-fermion unification.

5. Relativistic covariance - once the particle or string wave function is known, the Bohmian equations of motion and the corresponding probabilistic predictions are well defined and relativistic covariant.

Of course, string theory, supersymmetry, and boson-fermion unification are still not experimentally confirmed, so other variants of the Bohmian interpretation are not excluded by our theoretical results. Nevertheless, from an aesthetical point of view, such a unified Bohmian picture seems to be a very natural one. In addition, as both superstring theory and Bohmian mechanics contain some nonlocal features that may manifest as a preferred foliation of spacetime, it is possible that there is an even deeper not yet discovered relation between these two theories. Therefore, we believe that our results show that physicists interested in the Bohmian interpretation should become more interested in superstring theory. Conversely, we also believe that the results of this paper, together with those of [15, 14], suggest that physicists interested in string theory should become more interested in Bohmian mechanics as well. The fact that both theories are often unjustifiably criticized for not being experimentally testable is not the only thing that relates them. Although the fundamental problem of measurement in QM is traditionally not regarded as a problem that the "theory of everything" is supposed to solve, our results on the relation between superstring theory and Bohmian mechanics suggest that the solution of the measurement problem could also be a part of it.

\section{Acknowledgement}

The author is grateful to the referees for their valuable suggestions and stimulating objections. This work was supported by the Ministry of Science of the Republic of Croatia 
under Contract No. 098-0982930-2864.

\section{References}

[1] S. J. Gates Jr, M. T. Grisaru, M. Rocek and W. Siegel, Front. Phys. 58, 1 (1983); hep-th/0108200.

[2] B. Zwiebach, A First Course in String Theory (Cambridge University Press, Cambridge, 2004).

[3] M. B. Green, J. H. Schwarz and E. Witten, Superstring Theory (Cambridge University Press, Cambridge, 1987).

[4] J. Polchinski, String Theory (Cambridge University Press, Cambridge, 1998).

[5] J. D. Bjorken and S. D. Drell, Relativistic Quantum Mechanics (McGraw-Hill, New York, 1964).

[6] D. Bohm, Phys. Rev. 85, 166 (1952); Phys. Rev. 85, 180 (1952)

[7] D. Bohm and B. J. Hiley, Phys. Rep. 144, 323 (1987).

[8] D. Bohm, B. J. Hiley and P. N. Kaloyerou, Phys. Rep. 144, 349 (1987).

[9] P. R. Holland, Phys. Rep. 224, 95 (1993).

[10] P. R. Holland, The Quantum Theory of Motion (Cambridge University Press, Cambridge, 1993).

[11] J. S. Bell, Speakable and Unspeakable in Quantum Mechanics (Cambridge University Press, Cambridge, 1987).

[12] G. T. Horowitz, New J. Phys. 7, 201 (2005).

[13] N. Seiberg, hep-th/0601234.

[14] H. Nikolić, Eur. Phys. J. C 50, 431 (2007).

[15] H. Nikolić, Eur. Phys. J. C 47, 525 (2006).

[16] W. Struyve and H. Westman, quant-ph/0602229.

[17] D. Dürr, S. Goldstein, K. Münch-Berndl and N. Zanghì, Phys. Rev. A 60, 2729 (1999).

[18] G. Horton and C. Dewdney, J. Phys. A 35, 10117 (2002); G. Horton and C. Dewdney, J. Phys. A 37, 11935 (2004).

[19] H. Nikolić, Found. Phys. Lett. 17, 363 (2004).

[20] H. Nikolić, Found. Phys. Lett. 18, 123 (2005). 
[21] H. Nikolić, Eur. Phys. J. C 42, 365 (2005); H. Nikolić, Int. J. Mod. Phys. D 15, 2171 (2006), Honorable Mention of the Gravity Research Foundation 2006 Essay Competition.

[22] H. Nikolić, AIP Conf. Proc. 844, 272 (2006); quant-ph/0512065.

[23] N. D. Birell and P. C. W. Davies, Quantum Fields in Curved Space (Cambridge University Press, New York, 1982).

[24] J. D. Bjorken and S. D. Drell, Relativistic Quantum Fields (McGraw-Hill, New York, 1965).

[25] H. Nikolić, Found. Phys. 37, 1563 (2007).

[26] J. Polchinski, hep-th/9411028.

[27] N. Kemmer, Proc. Roy. Soc. A 173, 91 (1939).

[28] P. Ghose, D. Home and M. N. Sinha Roy, Phys. Lett. A 183, 267 (1993).

[29] W. Struyve, quant-ph/0506243.

[30] H. Nikolić, Phys. Lett. B 527, 119 (2002); H. Nikolić, Int. J. Mod. Phys. D 12, 407 (2003); H. Nikolić, Gen. Rel. Grav. 37, 297 (2005).

[31] L. H. Ryder, Quantum Field Theory (Cambridge University Press, Cambridge, 1984).

[32] S. S. Schweber, An Introduction to Relativistic Quantum Field Theory (Harper \& Row, New York, 1961).

[33] K. Berndl, D. Dürr, S. Goldstein and N. Zanghì, Phys. Rev. A 53, 2062 (1996).

[34] H. Nikolić, Found. Phys. Lett. 18, 549 (2005).

[35] D. Dürr, S. Goldstein, R. Tumulka and N. Zanghì, J. Phys. A 36, 4143 (2003).

[36] D. Dürr, S. Goldstein, R. Tumulka and N. Zanghì, Phys. Rev. Lett. 93, 090402 (2004).

[37] H. J. de Vega, J. R. Mittelbrunn, M. R. Medrano and N. Sánchez, Phys. Rev. D 52, 4609 (1995).

[38] H. Nikolić, arXiv:0705.3542.

[39] M. Daumer, D. Dürr, S. Goldstein and N. Zanghì, quant-ph/9601013.

[40] S. Goldstein, J. Taylor, R. Tumulka and N. Zanghì, J. Phys. A 38, 1567 (2005).

[41] E. C. G. Stückelberg, Helv. Phys. Acta 14, 322 (1941); Helv. Phys. Acta 14, 588 (1941).

[42] L. P. Horwitz, C. Piron, Helv. Phys. Acta 46, 316 (1973).

[43] A. Kyprianidis, Phys. Rep. 155, 1 (1987). 
[44] J. R. Fanchi, Found. Phys. 23, 487 (1993).

[45] H. Nikolić, Int. J. Quantum Inf. 7, 595 (2009).

[46] H. Nikolić, arXiv:0904.2287.

[47] R. Tumulka, J. Statist. Phys. 125, 821 (2006).

[48] H. Nikolić, Found. Phys. 38, 869 (2008).

[49] H. Nikolić, Int. J. Mod. Phys. A 22, 6243 (2007).

[50] H. M. Wiseman, New J. Phys. 9, 165 (2007).

[51] D. Dürr, S. Goldstein and N. Zanghì, arXiv:0808.3324. 